\title{
Stretchable Transducers for Kinesthetic Interactions in Virtual Reality
}

\author{
Extended Abstract \\ Robert Shepherd \\ Cornell University \\ Jose Barreiros \\ Cornell University \\ Bryan Peele \\ Cornell University \\ Omer Shapira \\ NVIDIA Corporation \\ David Luebke \\ NVIDIA Corporation \\ Benjamin Mac Murray \\ Cornell University \\ Josef Spjut \\ NVIDIA Corporation
}
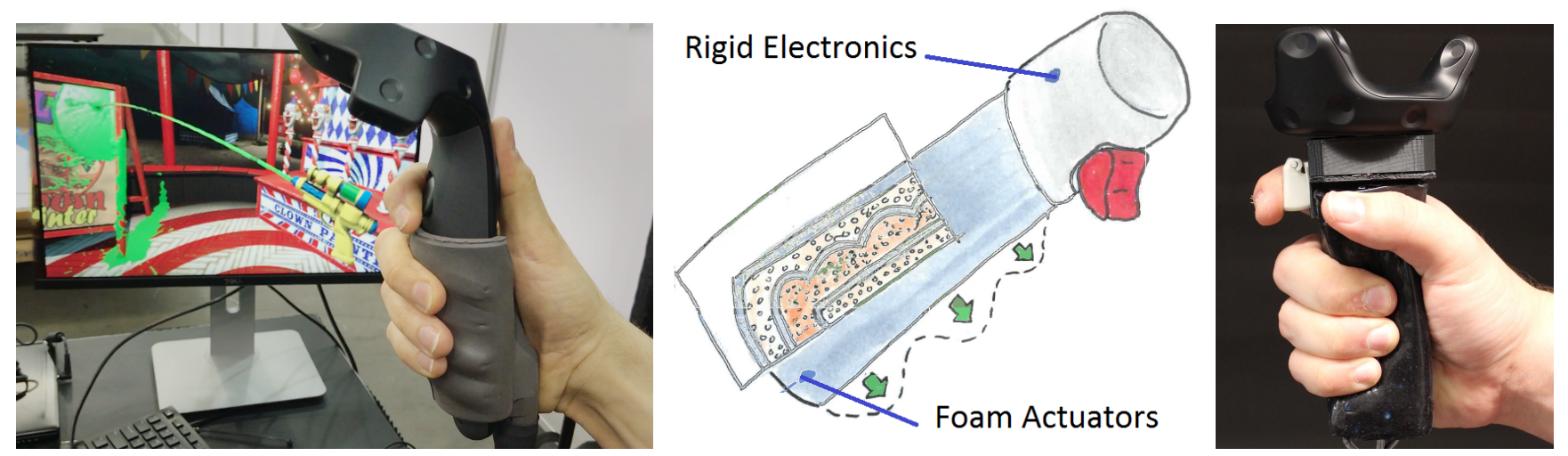

Figure 1: The tools of soft robotics enable immersive kinesthetic experiences in augmented and virtual reality while remaining safe to use. Left: Using fluidic elastomer actuators (FEAs), we demonstrate a soft skin that can provide force feedback to a motion tracked controller integrated with the VR Funhouse application. Middle and Right: A soft controller uses the variable compliance of soft structures to simulate different textures and materials.

\section{CCS CONCEPTS}

- Human-centered computing $\rightarrow$ Haptic devices; Virtual reality;

\section{KEYWORDS}

Human Computer Interaction, Haptics, Virtual Reality

\section{ACM Reference format:}

Robert Shepherd, Bryan Peele, Benjamin Mac Murray, Jose Barreiros, Omer Shapira, Josef Spjut, and David Luebke. 2017. Stretchable Transducers for Kinesthetic Interactions in Virtual Reality. In Proceedings of SIGGRAPH '17 Emerging Technologies, Los Angeles, CA, USA, July 30 - August 03, 2017, 2 pages.

https://doi.org/10.1145/3084822.3091103

Permission to make digital or hard copies of part or all of this work for personal or classroom use is granted without fee provided that copies are not made or distributed for profit or commercial advantage and that copies bear this notice and the full citation on the first page. Copyrights for third-party components of this work must be honored. For all other uses, contact the owner/author(s).

SIGGRAPH '17 Emerging Technologies, fuly 30 - August 03, 2017, Los Angeles, CA, USA

(C) 2017 Copyright held by the owner/author(s).

ACM ISBN 978-1-4503-5012-9/17/07.

https://doi.org/10.1145/3084822.3091103

\section{OVERVIEW}

Commercial virtual reality devices such as the Oculus Rift and HTC Vive enable experience designers to emulate a number of human sensory inputs with computer simulations. Commercial experiences have demonstrated plausibly realistic audiovisual sensory input, but somatosensory feedback has been more limited in scope. Most successful attempts in providing feedback to the human Kinesthetic system were considered power-demanding, expensive and potentially harmful to users, therefore somatosensory input has largely been addressed by vibration-based devices, like Linear Resonant Actuators and Eccentric Rotating Mass actuators, aimed at stimulating receptors near the surface of the skin. This method is widely accepted as a proxy in lieu of resisting to muscle tension, but it is not considered a path to realistic input.

Stemming from advances in materials science, the field of soft robotics constructs stretchable actuators, sensors and displays [ $\mathrm{Li}$ et al. 2016; Mac Murray et al. 2015; Zhao et al. 2016] using structures and materials that can be deformed at forces exerted by human muscles. To date, most demonstrations of soft robotics have focused on mobile robots, compliant grippers, and biomedical applications. Here we present the use of these technologies for kinesthetic feedback in virtual reality. The materials used to build these devices 
(e.g silicon) have mechanical properties similar to that of human tissue and are well-suited for applications that require direct and prolonged contact with human skin. Additionally, these systems are well-suited for commercial applications due to the low material cost and compatibility with scalable manufacturing techniques.

We provide two key demonstrations to highlight the use of fluidic elastomer actuators to provide haptic feedback. These demos allow users to progress through a series of brief experiences where the hand-held controller adjusts its form and behavior to match that of the virtual object used in each demo. The objects held in the demo include a goo gun, pistol and mallet.

\section{PNEUMATIC SKIN}

First, we have developed a thin ( $3 \mathrm{~mm}$ ) pneumatic skin to cover an HTC Vive controller. This pneumatic skin has 12 individually inflatable chambers placed in contact points similar to those of objects used in the game play of NVIDIA's VR Funhouse [NVIDIA 2016]. By sending impulses to the user based on actions in the game, the flow of gas into the chambers enhases the gameplay of VR Funhouse. For instance, pneumatic chambers along the bottom of the controller inflate to simulate the recoil of a revolver or the chambers undulate to simulate fluid moving through a water gun.

\section{FOAM CONTROLLER}

Our second demonstration is a rubber foam controller that changes stiffness and texture to represent a variety of objects from NVIDIA's VR Funhouse. The controller is composed of co-mingled blocks of foam that we selectively pressurize to provide force feedback upon gripping by the user. The combinations of stiffening different foam modules can represent a large number of Funhouse objects. For instance, the absence of pressurized blocks can simulate the palm area of a boxing glove, while a highly pressurized controller (all blocks pressurized) can mimic the stiff wooden handle of a mallet.

\section{REFERENCES}

Shuo Li, Bryan N Peele, Chris M Larson, Huichan Zhao, and Robert F Shepherd. 2016. A Stretchable Multicolor Display and Touch Interface Using Photopatterning and Transfer Printing. Advanced Materials 28, 44 (2016), 9770-9775.

Benjamin C Mac Murray, Xintong An, Sanlin S Robinson, Ilse M van Meerbeek, Kevin W O’Brien, Huichan Zhao, and Robert F Shepherd. 2015. Poroelastic foams for simple fabrication of complex soft robots. Advanced Materials 27, 41 (2015), 6334-6340. NVIDIA. 2016. NVIDIA VR Funhouse. http://store.steampowered.com/app/468700/. (2016). Accessed: 2017-03-20.

Huichan Zhao, Kevin O’Brien, Shuo Li, and Robert F Shepherd. 2016. Optoelectronically innervated soft prosthetic hand via stretchable optical waveguides. Science Robotics (2016). 